\title{
Possible Influence of the Ionosphere on the Impedance of a Ground-Based Antenna
}

\author{
James R. Wait
}

Contribution from Central Radio Propagation Laboratory, National Bureau of Standards, Boulder, Colo.

(Received April 12, 1962)

\begin{abstract}
The analysis for the impedance of a vertical electric dipole in the presence of an isotropic and homogeneous conducting half-space is presented. Various approaches to the problem are then briefly compared and some numerical results are presented in graphical form. The extensions to an anisotropic half-space are also considered. Finally, the dipole is located in the space between a homogeneous ground and a sharply bounded ionosphere. It is concluded that the presence of the ionosphere has a negligible effect on the impedance of a ground-based antenna unless the frequency is less than $1,000 \mathrm{c} / \mathrm{s}$ or so.
\end{abstract}

\section{Introduction}

The primary motivation for the present investigation was the idea that the ionosphere may influence the impedance of a ground-based antenna. Normally, one would expect the effect to be negligible since the separation between the ionosphere and ground is large compared with a wavelength for medium and higher radio frequencies. However, for lower frequencies, particularly those in the VLF and ELF regions, the height of the ionosphere is comparable with the wavelength. Thus, it would appear worthwhile to consider this question.

A related and more basic problem is the impedance of an antenna in the presence of a single boundary of separation between two media. Therefore, this problem is considered first.

\section{Formulation}

A vertical electric dipole of length $d s$ is located in air at height $z_{0}$ over a homogeneous half-space of refractive index $N$. The magnetic permeability of the whole space is $\mu$ and assumed to be a constant. With respect to a cylindrical coordinate system $(\rho, \phi, z)$, the interface is the plane $z=0$, the dipole is located at $z=z_{0}$ on the $z$ axis, the half-space $z>0$ is air with dielectric constant $\epsilon_{0}$, and the half-space $z<0$ is a homogeneous medium with (complex) dielectric constant $N^{2} \epsilon_{0}$. The situation is illustrated in figure 1a.

When the current in the dipole varies as $J_{0} e^{i \omega t}$ the fields can be found from the solution of a well-known boundary value problem [Sommerfeld, 1949]. The Hertz vector $\vec{\Pi}$ has only a $z$ component and, for $z>0$ is given by

$$
\Pi_{z}=\Pi_{2}^{(p)}+\Delta \Pi_{2}
$$

where the primary influence is

$$
\Pi_{z}^{(p)}=\frac{J_{0} d s}{4 \pi i \epsilon_{0} \omega} \frac{\exp \left[-i k\left[\rho^{2}+\left(z-z_{0}\right)^{2}\right]^{\frac{1}{2}}\right]}{\left[\rho^{2}+\left(z-z_{0}\right)^{2}\right]^{\frac{1}{2}}}
$$

and the secondary influence is

$$
\Delta \Pi_{z}=\frac{J_{0} d s}{4 \pi i \epsilon_{0} \omega} \int_{0}^{\infty} R(\lambda) J_{0}(\lambda \rho) \frac{e^{-2 u z_{0}}}{u} \lambda d \lambda
$$

where

$$
\begin{gathered}
R(\lambda)=\frac{u N^{2}-u_{1}}{u N^{2}+u_{1}}, \\
u=\left(\lambda^{2}-k^{2}\right)^{\frac{1}{2}}, u_{1}=\left(\lambda^{2}-N^{2} k^{2}\right)^{\frac{1}{2}}
\end{gathered}
$$

and

$$
k=\left(\epsilon_{0} \mu_{0}\right)^{\frac{1}{2}} \omega=2 \pi / \text { wavelength } .
$$

To satisfy the radiation conditions at infinity the real parts of $u$ and $u_{1}$ must be positive for $\lambda$ ranging from 0 to infinity. ${ }^{1}$

To carry out a complete calculation of the selfimpedance of the source dipole requires that the electric field in the gap on the connecting transmission line be determined from an integral equation formulation. In this general case it is necessary to consider the finite length of the antenna. However, if attention is confined to the change of the impedance resulting from the presence of the lower half space it is permissible to retain the dipole approximation.

By definition the impedance change $\Delta Z$ is related to the impedance $Z$ by

$$
\Delta Z=Z-Z_{0}
$$

${ }_{1}^{1}$ It may be remarked that if the integration contour is deformed the real parts of $u$ or $u_{1}$ can become negative. This appears to be the source of much confusion on the subject. For the present, the contour is the real axis of $\lambda$ and the real parts: of $u$ are positive. 
where

$$
\left.Z_{0}=Z\right]_{z_{0}=\infty}
$$

is the free space impedance. It is assumed that $Z_{0}$ is either known or can be separately accounted for. According to the "EMF method" it follows that

$$
\left.\Delta Z=\frac{\Delta E_{z} d s}{J_{0}}\right]_{\substack{z \rightarrow z_{0} \\ \rho \rightarrow 0}}
$$

where the limits are taken after performing the operation

$$
\Delta E_{z}=\left(k^{2}+\frac{\partial^{2}}{\partial z^{2}}\right) \Delta \Pi_{z}
$$

This leads readily to the result

$$
\Delta Z=-\frac{(d s)^{2}}{4 \pi i \epsilon_{0} \omega} \int_{0}^{\infty} R(\lambda) \frac{\lambda^{3}}{u} e^{-u \alpha} d \lambda
$$

where $\alpha=2 z_{0}$. It is convenient to normalize this by writing

$$
\Delta Z=R_{0} I
$$

where $R_{0}=20 k^{2}(d s)^{2}$ is the real part of $Z_{0}$ and is the radiation resistance of a dipole in the free space, and $I$ is then a dimensionless parameter which contains the essence of the problem.

\section{Asymptotic Approximation}

To obtain an asymptotic approximation to the integral for large values of $\alpha$ it is permissible to expand $R(\lambda)$ about $\lambda=0$ in a series of the form

$$
R(\lambda)=R(0)+\lambda R^{\prime}(0)+\frac{\lambda^{2}}{2} R^{\prime \prime}(0)+\frac{\lambda^{3}}{6} R^{\prime \prime \prime}(0)+\ldots
$$

where the prime indicates a differentiation with respect to $\lambda$ before setting $\lambda=0$. As it happens, in this particular case, $R^{\prime}(0), R^{\prime \prime \prime}(0)$, and all oddordered differentiated terms vanish. The integrals, to contend with, are then of the form

$$
P_{2 m}=\int_{0}^{\infty} \frac{\lambda^{2 m+1}}{u} e^{-u \alpha} d \lambda \text { for } m=1,2,3, \ldots
$$

Now

$$
P_{0}=\int_{0}^{\infty} \frac{e^{-u \alpha}}{u} \lambda d \lambda=\frac{e^{-i k_{\alpha}}}{\alpha}
$$

and, noting that $\lambda^{2}=u^{2}+k^{2}$, it readily follows that

$$
P_{2 m}=\left(k^{2}+\frac{\partial^{2}}{\partial \alpha^{2}}\right)^{m} \frac{e^{-i k_{\alpha}}}{\alpha} .
$$

This results in the expansion

$$
\begin{aligned}
I=\frac{\Delta Z}{R_{0}}= & -R(0)\left(k^{2}+\frac{\partial^{2}}{\partial \alpha^{2}}\right) e \frac{-i k_{\alpha}}{\alpha} \\
& -\frac{R^{\prime \prime}(0)}{2 !}\left(k^{2}+\frac{\partial^{2}}{\partial \alpha^{2}}\right)^{2} \frac{e^{-i k \alpha}}{\alpha} \\
& -\frac{R^{\mathrm{Iv}}(0)}{4 !}\left(k^{2}+\frac{\partial^{2}}{\partial \alpha^{2}}\right)^{4} \frac{e^{-i k_{\alpha}}}{\alpha}
\end{aligned}
$$

and so on. Retaining just the first two terms, this may be explicitly written

$I \cong-\left[3|R(0)| A e^{\left.i \arg R(0)-\phi_{a}-k \alpha\right]}\right.$

where

$$
\left.+6\left|k^{2} R^{\prime \prime}(0)\right| B e^{i\left[\arg R^{\prime \prime}(0)-\phi_{b}-k \alpha\right]}\right]
$$

$$
\begin{gathered}
A e^{-i \phi_{a}}=\left(1-\frac{i}{k \alpha}\right) \frac{1}{(k \alpha)^{2}}, \\
B e^{-i \phi_{b}}=\left[\frac{3}{(k \alpha)^{2}}-i\left(\frac{3}{(k \alpha)^{3}}-\frac{1}{k \alpha}\right)\right] \frac{1}{(k \alpha)^{2}}, \\
R(0)=\frac{N-1}{N+1} \\
k^{2} R^{\prime \prime}(0)=-\frac{2}{N} \frac{N-1}{N+1}=-\frac{2}{N} R(0) .
\end{gathered}
$$

A question immediately arises as to the validity of asymptotic expansions of this kind. In the present problem there is a pole where the denominator of $R(\lambda)$ is zero. This occurs when

$$
N^{2}\left(\lambda^{2}-k^{2}\right)^{\frac{1}{2}}+\left(\lambda^{2}-N^{2} k^{2}\right)^{\frac{1}{2}}=0 .
$$

Also, there are branch points at $\lambda= \pm N k$. To study the influence of these singular points the integral $P_{2 m}$ is written in the equivalent form

$$
P_{2 m}=\int_{C_{0}} \lambda^{2 m} e^{-u_{\alpha}} d u
$$

where the contour $C_{0}$, in the $u$ plane, is sketched in figure $1 \mathrm{~b}$ by the solid curve. The poles of the integrand, which occur when $N^{2} u+u_{1}=0$, are denoted by $\pm u_{p}$ and the branch points, which occur at $u=$ $\pm \sqrt{N^{2}-1} k$, are denoted $\pm u_{b}$. The location of the singularities in the complex $u$ plane are representative for a highly conducting medium where

$$
N \cong|N| e^{-i \pi / 4} \text { and }\left|N^{2}\right|>>1 .
$$

Now the asymptotic evaluation of the integral by a steepest descent method amounts to deforming the contour $C_{0}$ to contour $C_{1}$ shown in figure 1 . Here, $C_{1}$ is a straight line running parallel to the real axis and is defined by the relation $u=i k+x$ where $x$ runs from 0 to $\infty$ through real values.

It is evident from figure $1 \mathrm{~b}$ that no poles or other singularities are crossed in the deformation of the original contours to the steepest descent contour. 


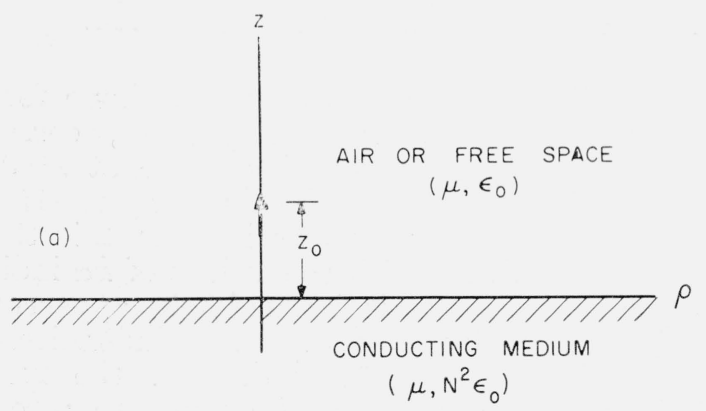

Figure 1a. Dipole over conducting half-space.

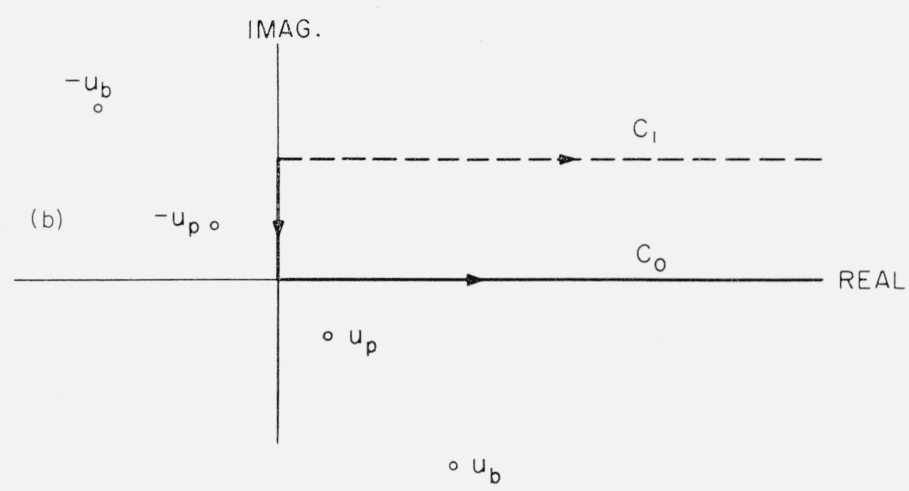

Figure 1b. The complex u plane.
Such is always the case for media in which the real part of $N^{2}>1$. In the case of certain plasma media this condition may be violated and then the integral along $C_{0}$ is asymptotically equal to the integral along $C_{1}$ plus $2 \pi i$ times the residue of the pole at $u_{p}$. In this study, it is assumed that the real part of $N^{2}>1$ so that the pole contribution does not appear in the asymptotic evaluation of the integral $P_{2 m}$.

\section{Numerical Integration Approach}

Unfortunately, in many cases of interest, the asymptotic series development for the integral is poorly convergent. Such is the case when $|N|$ is not reasonably large with respect to unity. In this case, it appears that a numerical integration is in order. After some study, the following method was employed. A new variable $g$, defined by $\lambda=k g$ is introduced. Then $I$ can be expressed in the form

$$
I=\int_{0}^{\infty} \frac{P(g)}{(g-1)^{\frac{1}{2}}} d g
$$

where

$$
P(g)=i \frac{3}{2} R(k g) g^{3}(1+g)^{-\frac{1}{2}} \exp \left[-k \alpha\left(g^{2}-1\right)^{\frac{1}{2}}\right]
$$

and

$$
R(k g)=\frac{N^{2}\left(g^{2}-1\right)^{\frac{1}{2}}-\left(g^{2}-N^{2}\right)^{\frac{1}{2}}}{N^{2}\left(g^{2}-1\right)^{\frac{1}{2}}+\left(g^{2}-N^{2}\right)^{\frac{1}{2}}}
$$

To comply with radiation conditions it is necessary to choose the square roots so that

and

$$
\operatorname{Lim}_{g \rightarrow \infty}\left(g^{2}-1\right)^{\frac{1}{2}} \rightarrow g
$$

$$
\operatorname{Jim}_{g \rightarrow \infty}\left(g^{2}-N^{2}\right)^{\frac{1}{2}} \rightarrow g
$$

Also

$$
\operatorname{Lim}_{g \rightarrow 0}\left(g^{2}-1\right)^{\frac{1}{2}} \rightarrow(g-1)^{\frac{1}{2}} \rightarrow i
$$

and

$$
\operatorname{Lim}_{g \rightarrow 0}\left(g^{2}-N\right)^{\frac{1}{2}} \rightarrow i N .
$$

To simplify the integration further the interval is broken into parts such that

$$
I=-i \int_{0}^{1} P(g)(1-g)^{-\frac{1}{2}} d g+\int_{1}^{\infty} P(g)(g-1)^{-\frac{1}{2}} d g .
$$

These integrals are not particularly suitable for numerical integration since there are singularities at $g=1$. However, by further change of variable in each integral this objection can be removed. In the first integral, we set

$$
x=\sqrt{g-1}
$$

and in the second integral,

$$
y=\sqrt{1-g} .
$$

Therefore,

$$
\frac{\Delta Z}{R_{0}}=I=2 \int_{0}^{\infty} P\left(1+x^{2}\right) d x-2 i \int_{0}^{1} P\left(1-y^{2}\right) d y
$$

where the function $P$ is defined by eq (23). These integrals are quite suitable for numerical or graphical integration.

\section{Compensation Theorem Approach}

Another approach to the determination of $\Delta Z$ is based on the compensation theorem from network theory. This method has been used extensively in the study of antenna ground systems [Wait and Pope, 1955; Maley and King, 1961]. Using such an approach one may write

where

$$
\Delta Z=\Delta Z]_{h=\infty}+\Delta Z_{t}
$$

$$
\Delta Z_{t}=-\frac{1}{J_{0}^{2}} \int_{0}^{\infty} H_{\phi}^{\infty}(\rho, 0) E_{\rho}(\rho, 0) 2 \pi \rho d \rho
$$

where $H_{\phi}^{\infty}(\rho, 0)$ is the magnetic field of the dipole on a perfectly conducting ground plane at $z=0$ and 
$E_{\rho}(\rho, 0)$ is the actual tangential electric field on the interface $z=0$. While this formula is exact, it is not a solution to the problem since $E_{\rho}(\rho, 0)$ is an unknown. However, if $|N|$ is reasonably large compared with unity,

$$
E_{\rho}(\rho, 0) \cong-\frac{120 \pi}{N} H_{\phi}^{\infty}(\rho, 0) .
$$

Inserting this result into eq (28) readily leads to

$$
\frac{\Delta Z}{R_{0}} \cong \frac{3}{N}\left[\frac{i}{k \alpha}\left(1-\frac{i}{k \alpha}\right) e^{-i k \alpha}-E i(-i k \alpha)\right]
$$

where

$$
E i(-i k \alpha)=-\int_{-i k \alpha}^{\infty} \frac{e^{-x}}{x} d x
$$

is the exponential integral. For computational purposes it is convenient to use the relation.

$$
E i(-i k \alpha)=C i(k \alpha)+i[(\pi / 2)-S i(k \alpha)]
$$

where $C i$ and $S i$ are the cosine and sine integrals which are extensively tabulated.

As an interesting check one may use the expansion

$E(-i x) \cong \frac{e^{-i x}}{-i x}\left[1+\frac{1}{(-i x)}+\frac{2 !}{(-i x)^{2}}+\frac{3 !}{(-i x)^{3}}+\ldots\right]$

to show, from eq (30), that

$\frac{\Delta Z_{t}}{R_{0}} \cong \frac{6 e^{-i k_{\alpha}}}{N}\left[\frac{1}{(k \alpha)^{2}}+\frac{i}{(k \alpha)^{3}}+\right.$ terms in $\frac{1}{(k \alpha)^{4}}$, etc. $]$

This result may be compared with the first two terms of the asymptotic expansion given by $\mathrm{eq}$ (15). Noting that

$$
\left[\frac{\Delta Z}{R_{0}}\right]_{N=\infty}=-3\left(1-\frac{i}{k \alpha}\right) \frac{1}{(k \alpha)^{2}} e^{-i k_{\alpha}}=\Delta Z^{(\infty)}
$$

and

$$
R(0)=\frac{N-1}{N+1} \simeq 1-\frac{2}{N}
$$

it immediately follows, from eqs (16) and (17), that the asymptotic result is

$$
\begin{aligned}
\Delta Z_{t}=\Delta Z-\Delta Z^{(\infty)} \cong \frac{6}{N} & {\left[\frac{1}{(k \alpha)^{2}}-\frac{i}{(k \alpha)^{3}}+\ldots\right] e^{-i k_{\alpha}} } \\
& +\frac{12}{N}\left[\frac{i}{(k \alpha)^{3}}+\ldots\right] e^{-i k \alpha}
\end{aligned}
$$

or

$\Delta Z_{t} \cong \frac{6 e^{-i k_{\alpha}}}{N}\left[\frac{1}{(k \alpha)^{2}}+\frac{i}{(k \alpha)^{3}}+\right.$ terms in $\frac{1}{(k \alpha)^{4}}$, etc. $]$

which to the order given is identical to the result obtained from the method based on the compensation theorem.

\section{Discussion of Numerical Results}

Some numerical results based on the above formulas are now considered. For $|N|=\infty$, corresponding to a perfectly conducting plane at $z=0$, the impedance increment is given exactly by eq (15). The results for this case are shown in figure 2 in the form of an Argand plot with the values of $k \alpha$ indicated on the curves. It is immediately apparent that the impedance increment becomes indefinitely large as $k \alpha$ becomes small. However, the real part of $I$ approaches unity as $k \alpha$ tends to zero. ${ }^{2}$

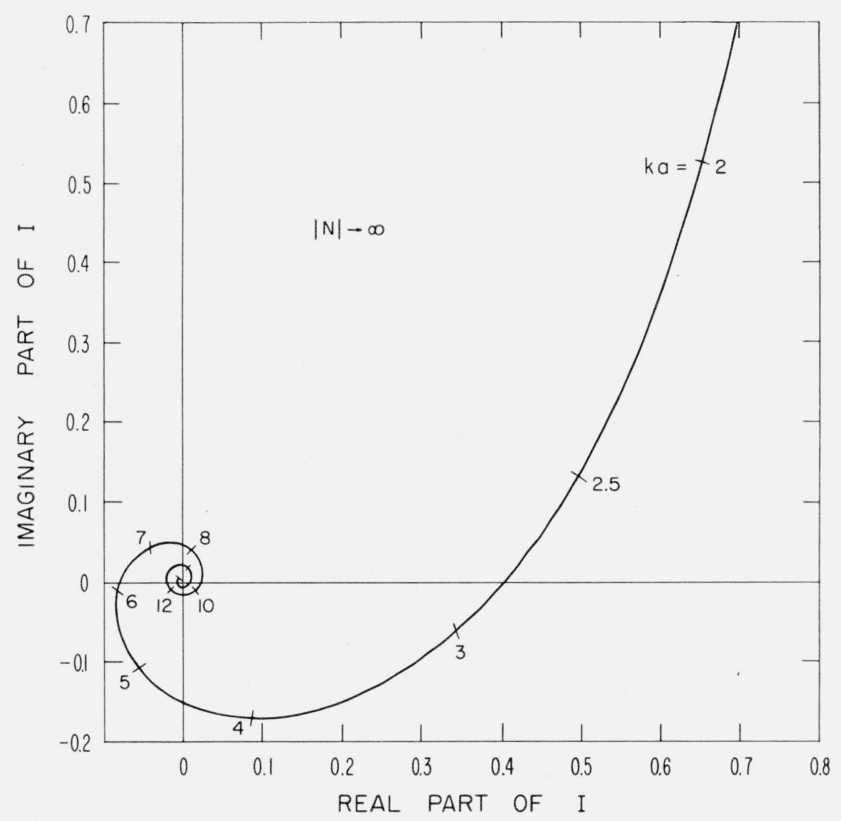

Figure 2. The change of impedance of a vertical dipole over a horizontal perfectly conducting plane.

(The results are expressed as a ratio to the free-space radiation resistance.)

This is physically acceptable and represents the doubling of the free-space radiation resistance as the dipole approaches the conducting plane.

Some results for finite values of $N$ are shown in figures $3 \mathrm{a}$ and $3 \mathrm{~b}$ for $N=30 e^{-i \pi / 4}$ and $10 e^{-i \pi / 4}$, respectively. These would correspond to a highly conducting half-space where displacement currents are negligible. The solid curves are based on using two terms in the asymptotic formula for $I$ given by eq (15). The dashed curves correspond to just using the first term in the same formula (i.e., set $B=0$ ). The distance between these curves (measured on a radius from the origin) gives some idea as to the accuracy of the two-term asymptotic formula for $I$. In the case of $|N|=30$, it appears that the results should be quite accurate even for

2 This follows from the relation

$$
\operatorname{Lim}_{x \rightarrow 0}\left\{\operatorname{Re}\left[-\frac{3}{x^{2}}\left(1-\frac{i}{x}\right) e^{-i x}\right]\right\}=1 .
$$




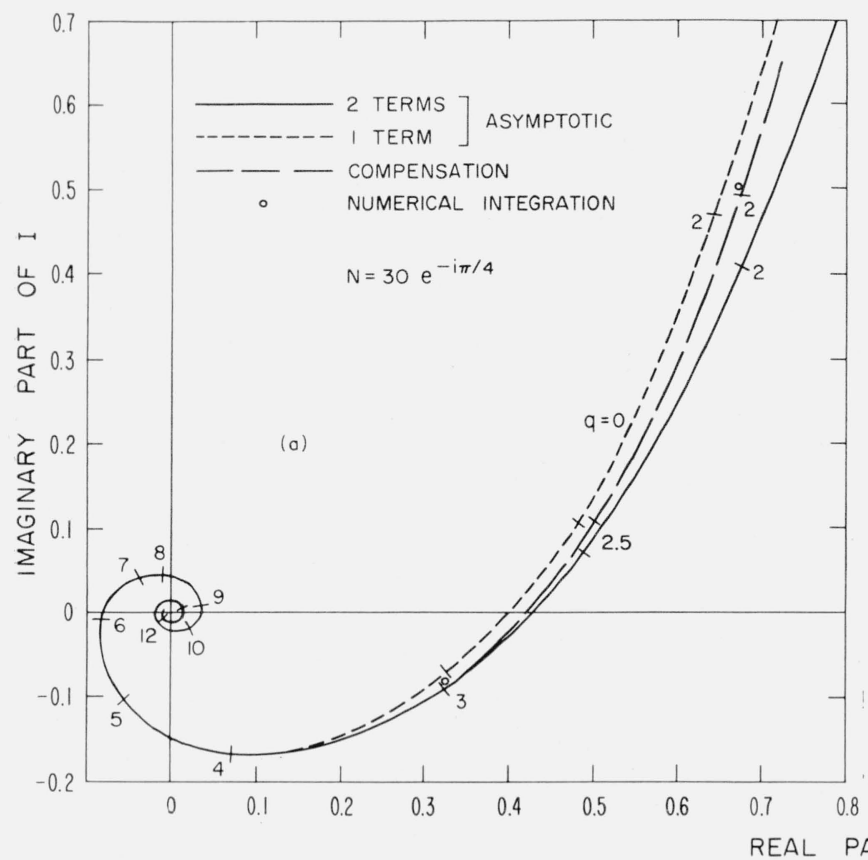

FIgURE 3a. The change of impedance of a vertical dipole over a horizontal imperfectly conducting plane.

(The results are expressed as a ratio to the free-space radiation resistance.)

relatively small values of $k \alpha$. A further check for these two cases is afforded by a numerical integration for $k \alpha=3$ for each case. This is shown by a small circle in both figure $3 \mathrm{a}$ and figure $3 \mathrm{~b}$.

The formula for $\Delta Z$ or $I$ based on the compensation theorem is also shown in figures $3 \mathrm{a}$ and $3 \mathrm{~b}$ and indicated by broken curves. In both cases it falls in between the curves for the one- and two-term asymptotic formulas. Also, it appears to agree quite closely with the points obtained from a direct numerical integration.

\section{Extension to an Anisotropic Half-Space}

An important extension of the preceding results is to a vertical electric dipole over an anisotropic half-space. In this case the dielectric constant is in the form of a tensor and the problem becomes quite complicated. However, if the anisotropic medium is a dense ionized medium with a superimposed d-c magnetic field parallel to the $z$ axis, symmetry about this axis is preserved. In this case, it is permissible to employ the Q.L. (quasi-longitudinal) approximation. The refractive index $\mu$ in this case is double-valued and given by

$$
\mu^{2} \cong 1-i \frac{\omega_{r}}{\omega} e^{ \pm i \tau}
$$

where

$$
\tan \tau=\omega_{L} / \nu, \omega_{r}=\omega_{0}^{2}\left(\nu^{2}+\omega_{L}^{2}\right)^{-\frac{1}{2}} .
$$

In the above, $\omega_{0}=$ angular plasma frequency,

$$
\nu=\text { collision number, assumed con- }
$$$$
\text { stant, and }
$$

$\omega_{L}=$ angular gyrofrequency .

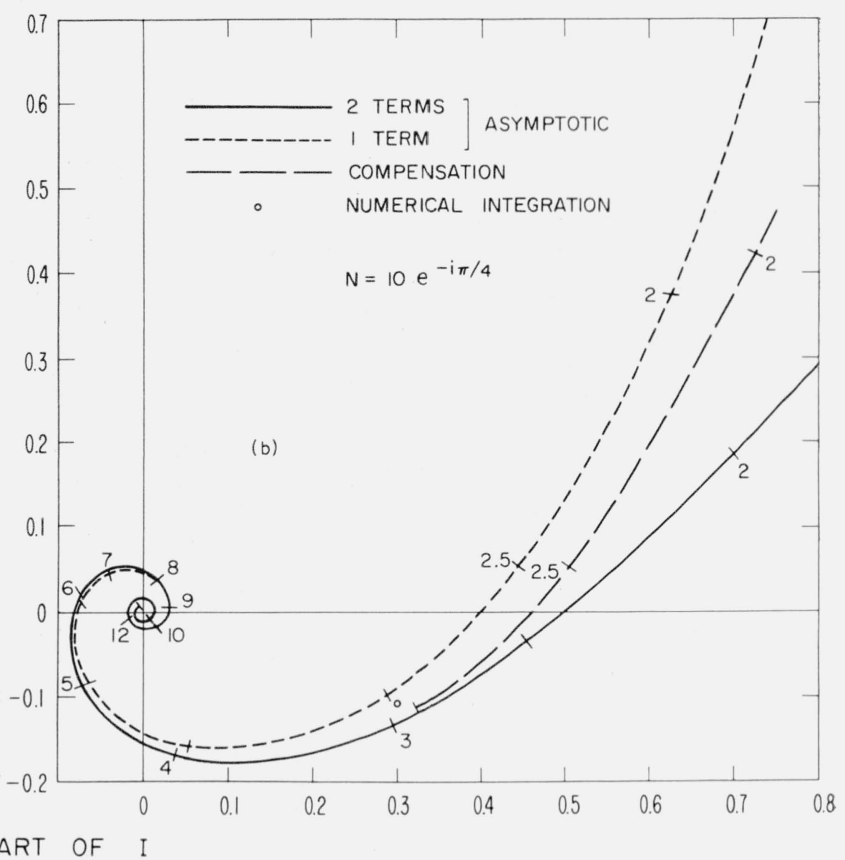

Figure 3b. The change of impedance of a vertical dipole over a horizontal imperfectly conducting plane.

(The results are expressed as a ratio to the free-space radiation resistance.)

It is also assumed that in writing these expressions, $\omega<<\nu$. A good discussion of the validity of the Q.L. approximation has been given by Budden [1961].

The incremental electric Hertz vector in the air space $z>0$ is written in the following form

$$
\Delta \Pi_{z}=\frac{J_{0} d s}{4 \pi i \epsilon_{0} \omega} \int_{0}^{\infty} \hat{R}(\lambda) J_{0}(\lambda \rho) \lambda u^{-1} d \lambda
$$

which has the same form as eq (8). However, in this case,

$$
\hat{R}(\lambda)=\frac{\left(\mu_{0}+\mu_{e}\right)\left(C^{2}-C_{0} C_{e}\right)+\left(\mu_{0} \mu_{e}-1\right)\left(C_{0}+C_{e}\right) C}{\left(\mu_{0}+\mu_{e}\right)\left(C^{2}-C_{0} C_{e}\right)+\left(\mu_{0} \mu_{e}+1\right)\left(C_{0}+C_{e}\right) C}
$$

where

$$
\begin{aligned}
C & =\left(1-S^{2}\right)^{\frac{1}{2}}, \quad S=\lambda / k \\
C_{e} & =\left[1-\left(S / \mu_{e}\right)^{2}\right]^{\frac{1}{2}} \\
C_{0} & =\left[1-\left(S / \mu_{0}\right)^{2}\right]^{\frac{1}{2}} .
\end{aligned}
$$

It can be readily verified that if the $d$-c magnetic field is removed (i.e., $\tau=0) \hat{R}(\lambda)$ reduces to $R(\lambda)$ given by eq (4) since $\mu_{0}=\mu_{e}=N$.

The incremental impedance $\Delta Z$ resulting from the presence of the anisotropic half-space is then formally given by

$$
\Delta Z=-\frac{(d s)^{2}}{4 \pi i \epsilon_{0} \omega} \int_{0}^{\infty} \hat{R}(\lambda) \lambda^{3} u^{-1} e^{-u_{\alpha}} d \lambda
$$

where again $\alpha=2 z_{o}$. The two-term asymptotic ex- 
pansion for this case can be written in the form

$$
\Delta Z=R_{0} I
$$

where

$I \cong-\left[3|\hat{R}(0)| A e^{i\left[\arg \hat{R}(0)-\phi_{a}-k \alpha\right]}\right.$

$$
\left.+6\left|k^{2} \hat{R}^{\prime \prime}(0)\right| B e^{i\left[\arg \hat{R^{\prime \prime}}(0)-\phi_{b}-k \alpha\right]}\right] .
$$

The complex quantities $A e^{-i \phi_{a}}$ and $B e^{-i \phi_{b}}$ are defined by eqs (16) and (17): Using eq (41) for $\hat{R}(\lambda)$ and carrying out the required differentiations leads to

$$
\begin{aligned}
\hat{R}(0) & =\frac{\mu_{0} \mu_{e}-1}{\mu_{0}+\mu_{e}+\mu_{0} \mu_{e}+1} \\
\hat{R}^{\prime}(0) & =0 \\
k^{2} \hat{R}^{\prime \prime}(0) & =-\frac{1}{2} \frac{\left(\mu_{0}+\mu_{e}\right)\left(2-\frac{1}{\mu_{e}^{2}}-\frac{1}{\mu_{0}^{2}}\right)}{\mu_{0}+\mu_{e}+\mu_{0} \mu_{e}+1} .
\end{aligned}
$$

Again, these reduce to the isotropic case when $\mu_{0}=$ $\mu_{e}=N$.

\section{Extension to Two-Boundary Case}

Another interesting extension is when the dipole is located in a homogeneous space bounded by two plane interfaces. The situation is illustrated in figure 4. The media beyond the plane interfaces (i.e., $z<0$ and $z>h$ ) are not specified at the moment. However, it is assumed that the fields are everywhere symmetrical about the polar axis.

For the situation described, and for $0 \leqq z \leqq z_{0}$ the electric Hertz vector has only a $z$ component and may be written in the form [Wait, 1960]

$$
\Pi_{z}=\frac{J_{0} d s}{4 \pi i \epsilon_{0} \omega} \int_{0}^{\infty} F(\lambda) J_{0}(\lambda \rho) \lambda u^{-1} d \lambda
$$

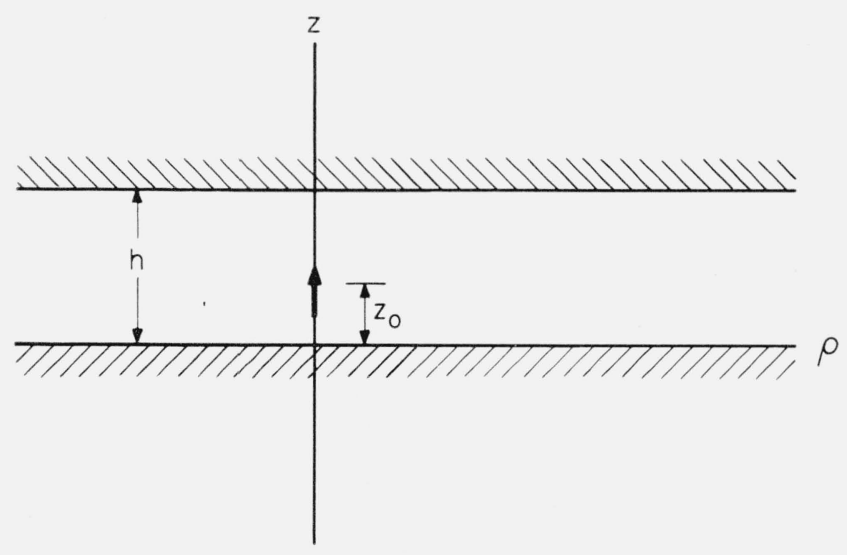

Figure 4. Dipole in homogeneous space bounded by two plane interfaces. where

$$
F(\lambda)=\frac{\left[e^{u z}+R_{1}(\lambda) e^{-u z}\right]\left[e^{u\left(h-z_{0}\right)}+R_{2}(\lambda) e^{-u\left(h-z_{0}\right)}\right]}{e^{u h}\left[1+R_{1}(\lambda) R_{2}(\lambda) e^{-2 u h}\right]} .
$$

If $z$ and $z_{0}$ are interchanged in the above expression, the result also holds for the range $z_{0} \leqq z \leqq h$. The coefficients $R_{1}$ and $R_{2}$ may be regarded as reflection coefficients for vertically polarized waves incident at a (complex) angle $\sin ^{-1}(\lambda / k)$ at the interfaces $z=0$ and $z=h$, respectively. When the two half-spaces are homogeneous media with refractive indices $N_{1}$ and $N_{2}$, the reflection coefficients have their usual Fresnel form given by

$$
R_{j}(\lambda)=\frac{u N_{j}^{2}-u_{j}}{u N_{j}^{2}+u_{j}} \quad \text { for } j=1,2
$$

and $u=\left(\lambda^{2}-k^{2}\right)^{\frac{1}{2}}$ and $u_{j}=\left(\lambda^{2}-k_{j}^{2}\right)^{\frac{1}{2}}$. In anisotropic cases $R_{1}$ and $R_{2}$ would be two-by-two matrices [Budden, 1961; Wait, 1960].

A special case worthy of some study is when the source dipole is on the bottom interface (i.e., $z_{0}=0$ ) and the lower half-space is a perfect conductor (i.e., $\left.\left|N_{1}\right|=\infty\right)$. For this case the incremental impedance $\Delta Z$ of the dipole is defined by

$$
\Delta Z=Z-[Z]_{h=\infty} .
$$

It follows without difficulty that

$$
\frac{\Delta Z}{2 R_{0}}=i \frac{3}{2} \int_{0}^{\infty} \frac{g^{3} R(k g) e^{-2 k h \sqrt{g^{2}-1}}}{\left[1-R(k g) e^{-2 k h \sqrt{g^{2}-1}}\right]} \frac{d g}{\sqrt{g^{2}-1}}
$$

where $2 R_{0}$ is the radiation resistance of the dipole located on a perfectly conducting plane. If one expands the denominator in a geometric series the formal connection between this result and eq (22) for the single boundary is evident. This integral has not been studied numerically. However, as a first approximation one may neglect multiple reflections between the two boundaries. This amounts to replacing the square bracket term in the denominator by unity. Then the formula for $\left(\Delta Z / 2 R_{0}\right)$ is the same as that given by the right-hand side of eq (15) if $\alpha$ is identified with $2 k h$.

\section{Concluding Remarks}

The question originally asked has been implicitly answered. Namely, the impedance of a groundbased antenna is not significantly influenced by the presence of the ionosphere unless the reflecting heights are somewhat less than a wavelength. For example, at a frequency of $15 \mathrm{kc} / \mathrm{s}$ it is seen from figures $2,3 \mathrm{a}$, or $3 \mathrm{~b}$ that the change of impedance (relative to the free space radiation resistance) is only of the order of one percent. This is hardly measurable. However, at $1,000 \mathrm{c} / \mathrm{s}, k \alpha$ is of the order of 4 and the relative change amounts to about 15 percent. This is measurable if special precautions were made. 
The important conclusion is that, in transmission loss calculations [Norton, 1959], the presence of the ionosphere may be neglected insofar as the resistance of the antenna is considered. Thus, the ionosphere only influences the propagation loss [Wait, 1959]. Such an assumption is implicitly made in most previous studies.

In a sequel to this paper the effect of raising the antenna to a large height will be investigated. Actually, the problem has already been formulated in this paper.

I thank Mrs. Alyce Conda for carrying out the computations. The work in this paper was supported by Electronics Research Directorate, Air Force Cambridge Research Laboratories, Contract PRO-61-568.

\section{References}

Budden, K. G., Radio waves in the ionosphere (Cambridge Univ. Press, Cambridge, 1961).

Maley, S. W., and R. J. King, The impedance of a monopole antenna with a circular conducting-disk ground system on the surface of a lossy half space, J. Research NBS 65D (Radio Prop.), No. 2, 183-188 (Mar.-Apr. 1961).

Norton, K. A., System loss in radio wave propagation, J. Research NBS 63D (Radio Prop.), No. 1, 53-73 (JulyAug. 1959).

Sommerfeld, A. N., Partial differential equations (Academic Press, New York, 1949).

Wait, J. R., Transmission of power in radio propagation, Electronic and Radio Engineer 36, No. 4, 146-150 (Apr. 1959).

Wait, J. R., Terrestrial propagation of very-low-frequency radio waves, J. Research NBS 64D (Radio Prop.), No. 2, 153-204 (Mar.-Apr. 1960).

Wait, J. R., and W. A. Pope, Input resistance of LF unipole aerials, Wireless Engineer 32, 131-138 (May 1955).

(Paper 66D5-216) 